Revista de Economia Política, vol. 30, $n^{\circ} 2$ (118), pp. 310-328 abril-junho/2010

\title{
Persistência inflacionária e curva de Phillips novo-keynesiana para o Brasil
}

FERNANDO DE AQUINO FONSECA NETO*

Inflation persistence and new Keynesian Phillips curves for Brazil. In this paper is shown that sustainable inflation persistence has theoretical support not only due price indexation, but also because of micro-foundations based on assumptions of Simon's bounded rationality and because of persistent mark-up shocks. the new keynesian phillips curve, estimated for brazil for the period 2000/2008, and the partial coefficients of determination for moving sub-periods of 36 months identifies inflation persistence as the main determinant of inflation, with the capacity gap presenting larger importance only in the end of the sample period. Inflation persistence requires harder monetary policy when neither accommodation is acceptable nor complementary policies in order to reduce it, such as the minimization of indexation mechanisms and control of the market power, are adopted.

Keywords: inflation persistence; bounded rationality; market power; expectations; Phillips curves.

JEL Classification: E31; D82; D84; E12.

\section{INTRODUÇÃO}

A taxa de inflação medida pelo Índice Nacional de Preços ao Consumidor Amplo (IPCA) após o encerramento da política de estabilização baseada em âncora cambial em fevereiro de 1999, apresentou média anual de 7,3\% a.a., de 1999 a 2007, reconhecendo-se a existência de vários choques, especialmente até 2002. No mesmo período, nenhum dos países do G7 — EUA, Japão, Alemanha, Reino Unido, França, Itália e Canadá — teve inflação ao consumidor média anual superior a 2,7\%, conforme dados divulgados no International Financial Statistics do Fundo Monetário Internacional (IFS/FMI). Com tais níveis de inflação, seus

\footnotetext{
"Banco Central do Brasil, e-mail: fernando.fonseca@bcb.gov.br. O autor isenta o Banco Central do Brasil de qualquer responsabilidade sobre o conteúdo do artigo. Submetido: Agosto de 2007; Aprovado: Janeiro 2009,
} 
impactos sobre o funcionamento da economia, tais como sobre as decisões de investimento e a distribuição de renda, podem até ser negligenciados, o que não seria consensual para o caso do Brasil. Nessas circunstâncias, é razoável que as autoridades econômicas no Brasil desejem reduzir as taxas de inflação para os padrões daqueles países.

Os benefícios esperados dessa redução, sobretudo em termos de melhoria das condições para os investimentos e de sustentação do poder de compra dos agentes sem mecanismos de proteção satisfatórios, são amplamente compartilhados. As políticas requeridas para alcançar tal redução é que estão longe de qualquer consenso. Alguns analistas acreditam que uma política monetária adequada e com credibilidade satisfatória manteria a inflação próxima de zero e o desemprego próximo de sua taxa natural, ainda que esse ajustamento possa ocorrer com alguma defasagem e venha a estar sujeito à ação de bolhas.

Em tal abordagem não há espaço para explicar a inflação por hiato do produto ou algum mecanismo de persistência. A inflação resultaria ou de choques de oferta - inflação de custos — ou de política monetária inadequada — inflação de demanda. O presente trabalho faz um levantamento de desenvolvimentos teóricos contrários a tais crenças a fim de contribuir para a identificação de possíveis procedimentos auxiliares, que possibilitem alcançar as metas para inflação com política monetária menos restritiva. As curvas de Phillips novo-keynesianas, por exemplo, ao considerar outras imperfeições de mercado além da ação de sindicatos, admitem a influência de outros fatores no comportamento da inflação.

Um ponto de partida seria a constatação de uma persistência inflacionária entre países com preços determinados pelo mecanismo de mercado, pelo menos nas últimas quatro décadas. Seria pouco plausível atribuí-la a políticas econômicas insuficientemente comprometidas com a estabilidade de preços em todos os países e em todo o período. Então, suas causas devem ser investigadas a partir das alterações na estrutura das economias. Nesse sentido, tem sido comum considerar tal persistência como inércia inflacionária. Entretanto, como a inflação inercial é tradicionalmente definida como um componente da inflação resultante da indexação de preços, no presente trabalho não trataremos a inércia como sinônimo de persistência, dado que esta última pode ser resultante também de outros fatores, tais como de padrões de comportamento compatíveis com as hipóteses de racionalidade limitada de Simon e de choques persistentes de mark-ups. No caso específico do Brasil após a política de estabilização baseada em âncora cambial, mesmo considerando a existência de indexação formal no preço de serviços públicos, e informal no caso da definição dos salários em algumas negociações coletivas, a persistência inflacionária em outros setores pode estar sendo sustentada por mecanismos complementares.

O trabalho está estruturado com a segunda seção trazendo comentários sobre teorias antecedentes que pretenderam explicar a persistência inflacionária. Em seguida, a terceira seção descreve os microfundamentos e as versões da curva de Phillips novo-keynesiana (CPNK) adotados como referenciais, que vêm a ser as abordagens mais recentes da persistência inflacionária. A quarta seção correspon- 
de à parte empírica do trabalho, onde são estimadas curvas de Phillips para o Brasil e coeficientes de determinação parcial em períodos móveis dos últimos 36 meses ao longo da amostra considerada para regressão. Na quinta seção realizamse comparações com outros estudos empíricos, enquanto na sexta seção são apresentadas as principais conclusões, assim como indicações para desdobramentos futuros da pesquisa.

\section{ABORDAGENS ANTECEDENTES}

Com base na chamada revolução das expectativas racionais, ocorrida para contestar a hipótese de expectativas adaptativas, um processo inflacionário só poderia subsistir em presença de política monetária inadequada ou com credibilidade insatisfatória. Vale salientar que desde o seu início essa abordagem tem sido objeto de críticas, podendo-se afirmar que a primeira crítica interna importante tenha sido formulada pelos chamados novos-keynesianos, ao atribuir à rigidez nominal e à falta de sincronia dos contratos a possibilidade de persistência da inflação, mesmo com a política monetária adequada e gozando de suficiente credibilidade.

Uma derivação representativa da CPNK com base em preços rígidos pode ser encontrada em Calvo (1983), cujo trabalho parte de três relações básicas. A primeira descreve o preço desejado da firma:

$$
{ }_{p}^{*}=p_{t}+\alpha y_{t}
$$

Onde: p* é o preço desejado;

p é o nível de preços da economia;

y é o produto, com o potencial normalizado para zero.

Uma vez que os próprios repasses de alterações de custos têm custos, como os de cardápio, tais repasses não ocorrem automaticamente, levando os preços praticados a divergir dos desejados. Nesse contexto, os preços praticados correspondem a uma média ponderada dos desejados, correntes e futuros, conforme expressão abaixo:

$$
x_{t}=\lambda \sum_{j=0}^{\infty}(1-\lambda)^{j} E_{t} p_{t+j}^{*}
$$

Onde x é o preço praticado;

$\lambda$ é uma taxa de decaimento para ponderação dos valores esperados dos preços desejados no futuro.

O nível geral de preços é determinado atribuindo-se pesos decrescentes com o tempo decorrido da fixação de cada preço vigente:

$$
p_{t}=\lambda \sum_{j=0}^{\infty}(1-\lambda)^{j} x_{t-j}
$$

A partir das expressões (1), (2) e (3), obtém-se a CPNK, com a inflação corrente determinada pelo hiato do produto e pela expectativa de inflação futura: 


$$
\Pi_{t}=\frac{\alpha \lambda^{2}}{(1-\lambda)} y_{t}+E_{t} \Pi_{t+1}
$$

Com relação aos fundamentos microeconômicos, em Mankiw (1985) argumenta-se que a rigidez de preços determinada pelos custos de cardápio é sustentável para firmas com algum poder de mercado, por acarretar perdas privadas apenas de segunda ordem, mesmo quando as perdas sociais são de primeira ordem. Uma evidência microeconômica que debilita a hipótese de custos de cardápio pode ser encontrada em Zbaracki et al. (2004) - um estudo empírico dos custos associados a alterações de preços em uma grande indústria. Naquele estudo de caso verifica-se que apenas uma pequena percentagem dos custos refere-se à impressão e distribuição de listas de preços, sendo muito mais importantes os custos relativos à obtenção de informações, tomadas de decisão, negociações e comunicações, potenciais geradores de rigidez de outra natureza - a rigidez de informações.

Uma sofisticação do debate foi alcançada ao se considerar a relevância do poder de mercado dos formadores de preço. Nessas condições, as interações estratégicas entre eles puderam ser analisadas com o instrumental da teoria dos jogos. Em Simonsen (1983), a inflação que persistiria à política monetária crível e voltada à estabilização de preços é explicada a partir de um jogo de formação de preços. Em tal jogo, o equilíbrio de Nash não é relevante para sua solução, pois as perdas de mercado que poderiam resultar de diferentes estratégias de concorrentes seriam, em geral, suficientes para que os formadores de preço adotem estratégias maxmin (que maximizam o retorno negativo do pior estado da natureza).

Outras restrições à hipótese de expectativas racionais têm vindo do comportamento empírico da inflação. Um fato marcante seria a evidência de mudança de comportamento da inflação, a partir do início dos anos setenta, em países que tradicionalmente mantém uma política monetária efetiva, como os do Gráfico 1.

\section{Gráfico 1: Níveis de preço no longo prazo}

IPC $(2007=100)$

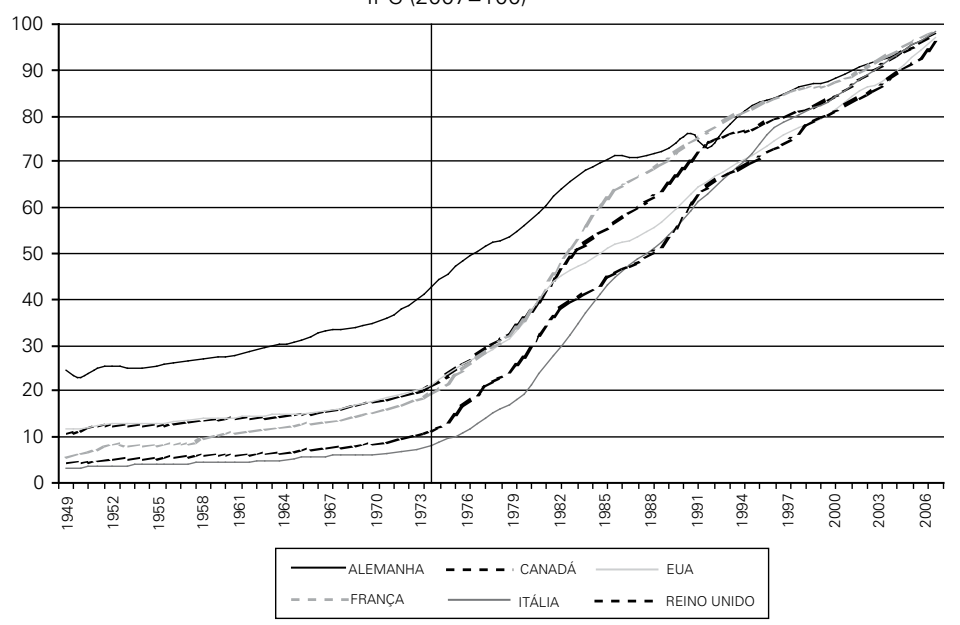


Em termos de modelagem estatística, pode-se considerar que no período anterior a inflação apresentava comportamento próximo de um ruído branco (white noise), enquanto no período atual ela seria mais bem modelada como um passeio aleatório (random walk) (Barsky, 1987; Ball, 2000), caracterizando o que se pode chamar de persistência inflacionária. Essa persistência faz da inflação passada um previsor satisfatório da inflação corrente, sendo consistente com o conceito de NAIRU — taxa de desemprego não-acelerante da inflação (nonaccelerating inflation rate of unemployment). Assim, de acordo com Ball e Mankiw (2002), o valor do conceito de NAIRU depende do regime de política monetária.

Observe-se que esse comportamento, de passeio aleatório, abrange pelo menos os países ocidentais mais desenvolvidos por mais de 30 anos, o que indica a possibilidade de uma mudança intrínseca o comportamento da inflação e não apenas um regime de política monetária que acomodaria essa persistência inflacionária. Num mundo em que a inflação se mantinha próxima de um ruído branco, a inflação esperada seria normalmente próxima de zero, levando a que a taxa natural de desemprego estivesse associada a preços estáveis. Em um regime de política monetária como o vigente nas economias de mercado desenvolvidas nas últimas décadas, argumenta-se naquele artigo que o conceito de NAIRU - que corresponde à taxa de desemprego associada à estabilidade da inflação - adquire relevância e pode ser considerado sinônimo da taxa natural de desemprego.

O que pode ser considerado uma tentativa de acomodar as observações empíricas sobre as relações entre inflação e desemprego com a tradição novo-clássica são modelos que incluem pequenos desvios na hipótese de expectativas racionais. Orphanides e Williams (2003) propõem um modelo em que os agentes possuem conhecimento imperfeito da estrutura da economia, levando-os a adotar um processo de aprendizagem perpétua, a partir do qual formam expectativas e atualizam continuamente suas crenças. A hipótese de expectativas racionais com aprendizagem tem sido considerada em uma série de trabalhos (Bullard \& Mitra, 2002; Collard \& Dellas, 2004; Milani, 2005, nos quais, sob determinadas condições, uma economia com aprendizagem converge para um equilíbrio com expectativas racionais. Entretanto, em um ambiente econômico em constante alteração as crenças não convergem para um equilíbrio, mas para uma distribuição ergódiga em torno de um equilíbrio com expectativas racionais (Evans \& Honkapohja, 2001). Portanto, modelos nessa direção podem admitir persistência da inflação e desemprego descolado de sua taxa natural, mesmo com política monetária adequada e em um ambiente com expectativas racionais.

Explicações alternativas têm surgido nos últimos anos para essa persistência inflacionária, estando as baseadas na hipótese de heterogeneidade das expectativas inflacionárias dentre as mais elaboradas. Quanto à fonte de tal heterogeneidade, alguns trabalhos atribuem à credibilidade imperfeita na política monetária (Ball, 1995); outros a agentes com racionalidade limitada — com regras de formação de preços que não satisfazem à hipótese de expectativas racionais (Amato \& Laubach, 2003); havendo ainda os que atribuem a heterogeneidade dessas expectativas 
à rigidez de informações, devido aos agentes econômicos não atualizarem essas expectativas continuamente, em função dos custos de adquirir as informações e realizar a re-otimização (Mankiw \& Reis, 2002). Embora com microfundamentos mais elaborados e mimetizando persistência inflacionária, esses modelos até muito recentemente não chegavam a reproduzir a persistência efetivamente observada nos processos inflacionários reais, que, longe de serem temporárias ou meramente respostas a choques na política monetária, têm um caráter endógeno e temporalmente sustentável.

Esse comportamento empírico remete ao que se denomina inércia inflacionária, componente da inflação resultante da indexação de preços — vinculação sistemática de preços correntes a variações defasadas de algum conjunto de preços. Nessa trilha, Christiano et al. (2005) propõem uma generalização do modelo de Calvo (1983), em que os preços são parcialmente indexados a índices agregados durante os intervalos randômicos de tempo entre as otimizações. Com tal hipótese chega-se a uma generalização da curva de Phillips na forma abaixo:

$$
\Pi_{t}-\rho \Pi_{t-1}=\frac{\alpha \lambda^{2}}{(1-\lambda)} y_{t}+E_{t}\left[\Pi_{t+1}-\rho \Pi_{t}\right]
$$

onde $0 \leq \rho \geq 1$ indica o grau de indexação, $\operatorname{com} \rho=0$ correspondendo ao modelo de Calvo (1983) e $\rho=1$ o caso de indexação integral. Essa versão da curva de Phillips tanto inclui a inflação defasada, que tem funcionado como principal termo para ajustamento de modelos empíricos a dados de inflação dos países com economia de mercado em geral, quanto gera uma persistência sustentável, como vem ocorrendo nos processos inflacionários reais. Entretanto, a versão da curva de Phillips expressa em (5) carrega forte limitação, qual seja, a observação de persistência inflacionária em economias sem evidências microeconômicas diretas de indexação significativa de preços. O que se tem observado em algumas economias com baixa inflação é que muitos preços permanecem inalterados em termos monetários por vários meses.

\section{REFERENCIAL TEÓRICO}

A aparente inconsistência entre a baixa indexação de preços em alguns países, em nível individual, e a importância da inflação passada na modelagem empírica da inflação, ${ }^{1}$ em nível agregado, tem motivado argumentações tanto de natureza econômica quanto estatística. Dentre as de ordem estatística, vale citar Cogley e Sbordone (2005), que realizaram uma log-linearização mais completa em torno da inflação zero, para gerar uma CPNK tradicional, obtendo uma aproximação mais sofisticada, que agrega termos adicionais e mais afastados no tempo de inflação

\footnotetext{
${ }^{1} \mathrm{O}$ papel central da inflação passada em análises empíricas de processos inflacionários está sistematizado em Gordon (1997).
} 
futura, a partir da tendência da taxa de inflação em cada período. Em exercícios empíricos, aqueles autores observaram alta correlação entre a tendência da taxa de inflação e termos defasados dessa mesma inflação, o que explicaria o bom ajustamento obtido com aqueles termos na curva de Phillips.

No mesmo sentido, Paloviita (2006), ao estimar curvas de Phillips com séries de expectativas inflacionárias, ao invés de assumir que as expectativas são racionais e utilizar valores futuros da própria série de inflação considerada, obteve reduções nos coeficientes dos termos defasados de inflação. Adicionalmente, Woodford (2007) indica trabalhos em que constam evidências de que quanto mais estável a taxa de inflação, menor a sua correlação com as expectativas inflacionárias.

Argumentações de natureza econômica para a aparente inconsistência entre as perspectivas macro e micro do comportamento da inflação podem partir de uma melhor discussão dos microfundamentos. Nesse sentido, é essencial retomar uma célebre dissidência ocorrida entre os precursores da hipótese de expectativas racionais. Herbert Simon, que chegou a ganhar o Prêmio Nobel de economia de 1978 precisamente por seus estudos sobre o processo de tomada de decisão dentro das organizações econômicas, desenvolveu pesquisas, até o início dos anos 1960, em colaboração com outros membros da Graduate School of Industrial Administration da Carnegie-Mellon University (Sent, 1997).

Tais pesquisas, que originalmente consistiam no desenvolvimento e aplicação de técnicas matemáticas para tomadas de decisão (Holt, Modigliani, Muth \& Simon, 1960), tiveram desdobramentos em duas direções distintas: a hipótese de expectativas racionais e a hipótese de racionalidade limitada. A primeira, formulada por Muth e tendo como maiores partidários Lucas e Sargent, teve grande êxito em formalizações matemáticas, bem como levaram a resultados bastante convenientes, sobretudo aos interesses dos mais poderosos, num período de predomínio de doutrinas favoráveis ao internacionalismo financeiro, que pode estar se esgotando com a "Crise do Subprime".

A hipótese de racionalidade limitada de Simon é proposta em contraste com o critério de otimização da teoria econômica tradicional. Os recursos materiais, mentais e de tempo requeridos pela otimização podem ser usados para outros fins ou mesmo não estar disponíveis ao agente, de modo que ele tende a preferir, ou tem que adotar, o desenvolvimento de alguma aspiração e buscar a sua satisfação. Este seria um padrão de comportamento mais próximo do empiricamente observado. Simon (1981) inclui como dois aspectos mais difíceis de tratar, mesmo com soluções subótimas, a incerteza em relação a eventos externos e a existência de expectativas mútuas, o que faz com que as instituições existam e funcionem.

Em Sargent (1993) uma proposta de conciliação entre as hipóteses de expectativas racionais e de racionalidade limitada é apresentada. Nessa proposta, a racionalidade limitada é definida com a inclusão de dois elementos ao modelo de otimização dos agentes econômicos: (i) informação incompleta ou imprecisa sobre as variáveis exógenas relevantes e o funcionamento da economia e (ii) capacidade de adaptação ou aprendizado. Essa definição de racionalidade limitada, contudo, mantém o processo de otimização, justamente o que a noção de Simon pretende 
superar. Assim, os agentes otimizam uma função objetivo interna (seu ambiente interno) adaptando-se às mudanças no ambiente externo, mesmo na presença de informação imprecisa ou incompleta. Portanto, a racionalidade limitada de Sargent não teria qualquer relação com a de Simon, que contesta a capacidade e a eficiência da realização da otimização pelo agente econômico em suas tomadas de decisão.

Os microfundamentos para a persistência inflacionária em geral podem ser considerados aplicações da hipótese de racionalidade limitada de Simon. Assim, o fato de grande parte dos preços permanecerem inalterados por vários meses, a despeito de alterações relevantes nas condições de custo e de demanda, pode ser abordado com ferramentas da teoria da informação. Nessa linha, Sims (2003) propõe hipótese de que os indivíduos têm capacidade limitada para absorver informações. Abordagem alternativa para explicar essa defasagem da nova otimização enfatiza os custos de se analisar as informações pertinentes. Begg e Imperato (2001) argumentam que pensar é trabalhoso, de modo que as pessoas não reavaliam seus planos sempre que o conjunto de informações relevante se altera. A rigor, o quanto uma pessoa pensa sobre um tema depende do benefício que acredita adquirir com isso. Indivíduos sem interesse específico em conjuntura econômica despenderão pouco tempo pensando sobre seus temas, embora algumas circunstâncias possam afetar tal alocação de seus recursos mentais, tais como a maior exposição daqueles temas na mídia.

Carroll (2001) identifica algumas evidências diretas da lenta disseminação de informações referentes à inflação. Comparando a expectativa de inflação de previsores profissionais e do público em geral, ele encontra que os primeiros fazem melhores previsões que o segundo. Um resultado interessante, contudo, foi que as expectativas do público respondem às expectativas dos profissionais com determinada defasagem. Adicionalmente, encontra-se que a divergência de expectativas entre profissionais e público, assim como a lentidão do ajustamento das expectativas do público, se reduz com o aumento das notícias e comentários sobre inflação.

Vale ressaltar que qualquer que seja a fonte de rigidez dos preços, seu ajustamento carregará uma dimensão temporal, resultando em persistência inflacionária, em termos macroeconômicos. Nesse sentido, tanto a rigidez de preços da CPNK tradicional quanto a rigidez de informações ou qualquer explicação plausível da ausência de otimização sempre imediata de preços, são hipóteses para a fundamentação microeconômica da persistência inflacionária. Contudo, modelos que associassem, formalmente, essa rigidez com uma persistência inflacionária com dinâmica própria, somente vieram a ser formulados em Mash (2006) e Sheedy (2007). Esses modelos podem ser definidos como CPNK generalizada, onde a CPNK tradicional, como proposta em Calvo (1983), seria um caso especial. Independente da fonte de rigidez dos preços, a sua ligação formal com uma persistência inflacionária sustentável foi efetuada mesmo sem indexação de preços, a partir da hipótese de relação positiva entre a idade do preço e sua probabilidade de ajustamento, conforme expressão abaixo: 


$$
\rho_{t}=\sum_{j=0}^{\infty} \theta_{j} x_{t-j}
$$

Onde p é o nível geral de preços, $\theta_{j}$ a proporção de firmas usando um preço fixado $j$ períodos atrás e $x$ corresponde ao preço ótimo intertemporal. Esta hipótese permite deduzir uma CPNK generalizada, em que a inflação é decomposta em um componente de persistência estrutural, cujo comportamento está associado aos próprios valores defasados, e outro componente determinado pelas expectativas de inflação, choques de oferta $(\mathrm{u})$ e hiato do produto $(\mathrm{y})$ :

$$
\Pi_{t}=\sum_{j=1}^{n-1} \gamma_{j} \Pi_{t-j}+\sum_{j=1}^{n} \gamma_{-j} E_{t} \Pi_{t-j}+\delta_{y} y_{t}+\delta_{u} u_{i}
$$

Portanto, chega-se a uma curva de Phillips formalmente derivada e com termos defasados da inflação consistentes com outros mecanismos além da indexação. Vale ressaltar que a relação macroeconômica expressa em (7) é compatível com qualquer fundamentação microeconômica para a rigidez de preços que não exclua a hipótese de probabilidade de ajustamento crescendo com a idade do preço. Nesse sentido, a expressão (7) representaria o caso geral, enquanto a CPNK tradicional, como derivada em Calvo (1983), seria um caso especial que se obtém com as probabilidades de ajustamento iguais para todos os preços.

Outra hipótese para a persistência inflacionária, também compatível com uma CPNK da forma expressa em (7), seria que a mesma resultaria de um processo de formação de preços na presença de mercados imperfeitos. Os formadores de preço com suficiente poder de mercado tentariam, recorrentemente, elevar e manter suas margens de lucro, desalinhando constantemente a configuração de preços e levando todos os formadores de preço a tentar repassar os consequentes aumentos de custos. Investigações empíricas em Neiss (2001), Cavelaars (2003), Smets e Wouters (2003) e Przybyla e Roma (2005) fornecem suporte à hipótese de que os graus de monopólio nos mercados é um dos determinantes dos diferenciais de inflação, tanto entre os países da OECD quanto entre os da União Europeia.

\section{ANÁLISE ECONOMÉTRICA}

A estimação empírica da expressão (7) foi efetuada a partir das seguintes séries mensais:

Inflação: variação percentual do Índice Nacional de Preços ao Consumidor Amplo (IPCA) Fonte: Instituto Brasileiro de Geografia e Estatística (IBGE), Sistema Nacional de Índices de Preços ao Consumidor (SNIPC)

Produto: Produção industrial da indústria geral — índice de quantum Fonte: IBGE, Pesquisa Industrial Mensal — Produção Física (PIM-PF) 
Utilização de Capacidade: utilização da capacidade instalada na indústria Fonte: Confederação Nacional da Indústria (CNI), Indicadores Industriais.

Taxa de câmbio: comercial, para venda, real (R\$) / dólar americano (US\$) média no período. Fonte: Banco Central do Brasil (BCB) SISBACEN/PTAX800.

Expectativas inflacionárias: Expectativas de mercado médias e medianas da variação do IPCA. Fonte: BCB, Pesquisa da Gerência-Executiva de Relacionamento com Investidores (GERIN)

As séries de expectativas inflacionárias utilizadas têm início para a inflação de abril de 2000, o que limita a amostra a ter início naquele mês. Para modelar o comportamento mensal da inflação, ${ }^{2}$ as seguintes variáveis foram consideradas:

Inflação mensal (IM) : Variação percentual mensal do IPCA.

Hiato do produto (HY): Razão entre série de produção industrial, dessazonalizada pelo método X12, e sua tendência, obtida com o filtro Hodrick-Prescott (HP).

Hiato de capacidade (HC): Razão entre a série de utilização de capacidade, dessazonalizada pelo método X12, e sua tendência, obtida com o filtro HodrickPrescott (HP).

Choques cambiais (CC): Variação percentual mensal da taxa de câmbio.

Expectativas inflacionárias médias (EMD): média das expectativas médias de variação mensal do IPCA dos cinco primeiros dias úteis do mês anterior. ${ }^{3}$

Expectativas inflacionárias medianas (EMN): média das expectativas medianas de variação mensal do IPCA dos cinco primeiros dias úteis do mês anterior.

Dummy para inflação de novembro de 2002 (D1102): para controlar pico inflacionário naquele mês.

Dummy política (DPOL): para controlar a inflação no período de novembro de 2002 a janeiro de 2003, que antecedeu o então novo governo.

A curva de Phillips novo-keynesiana para o Brasil foi estimada com os seguintes procedimentos:

(i) As taxas foram transformadas em fator ( $\mathrm{FT}=1+\mathrm{T} / 100)$, possibilitando as transformações logarítmicas necessárias para a estimação de modelos multiplicativos.

(ii) Aplicaram-se testes de raiz unitária de Phillips-Perron (PP) e de Dickey-Fuller aumentado (ADF) nas séries utilizadas, cujos resultados estão apresentados na Tabela 1, concluindo-se que todas podem ser consideradas estacionárias.

\footnotetext{
${ }^{2}$ Todos os procedimentos econométricos para este trabalho foram efetuados com o programa E-Views 6 .

${ }^{3}$ Observe-se que períodos mais distantes para a formação de expectativas inflacionárias mensais tendem a não incorporar suficiente interesse dos formadores, enquanto períodos mais próximos estariam contaminados por informações de outros índices de preços, inclusive do IPCA-15, com períodos de coleta parcialmente coincidentes.
} 
Tabela 1

\begin{tabular}{|l|c|c|c|c|}
\hline \multicolumn{5}{|c|}{ TESTES DE RAIZ UNITÁRIA } \\
\hline \multirow{3}{*}{ série } & \multicolumn{2}{|c|}{ Phillips-Perron (PP) } & \multicolumn{2}{c|}{ Dickey-Fuller Aumentado (ADF) } \\
\cline { 2 - 5 } FIM & especificação & estatística $\Delta$ & especificação & estatística $\Delta$ \\
FEMN & C & $-5,10^{*}$ & $\Delta y(t-1)$ a $\Delta y(t-2)$ e C & $-3,72^{*}$ \\
FEMD & $C$ & $-4,69^{*}$ & $\Delta y(t-1)$ a $\Delta y(t-12)$ & $-0,01$ \\
FCC & $C$ & $-4,39^{*}$ & $\Delta y(t-1)$ a $\Delta y(t-12)$ & $-0,00$ \\
HPI & $C$ & $-5,99^{*}$ & C & $-5,86^{*}$ \\
HUC & $C$ & $-8,39^{*}$ & $\Delta y(t-1)$ a $\Delta y(t-3)$ e C & $-3,88^{*}$ \\
RESID & $C$ & $-4,08^{*}$ & $\Delta y(t-1)$ a $\Delta y(t-3)$ e C & $-3,44^{*}$ \\
\hline
\end{tabular}

(iii) Os períodos de transmissão de alterações no hiato e de choques cambiais foram identificados, tentativamente, na especificação da regressão. Para tanto, em lugar de defasagens distribuídas, os efeitos defasados de cada variável foram concentrados em uma série, o que permite uma leitura mais direta dos resultados, em termos de elasticidade, resposta a choques, determinação parcial. Assim, o efeito de uma variável por k períodos foi especificado por:

$$
X(\mathrm{k})=\left(\prod_{\mathrm{j}=0}^{\mathrm{k}-1} X_{\mathrm{t}-\mathrm{j}}\right)^{1 / \mathrm{k}}
$$

(iv) A eventual presença de autocorrelação ou heterocedasticidade, embora não afete a consistência dos estimadores de mínimos quadrados ordinários, comprometem a consistência das estimativas de suas covariâncias. Nessas condições, adotou-se sempre o estimador para a covariância proposto em Newey e West (1987), que é consistente tanto na presença de heterocedasticidade quanto de autocorrelação de formas desconhecidas. Assim, corrigem-se as estimativas dos desvios-padrão, possibilitando a realização dos testes de significância para os estimadores.

(v) A seleção do melhor modelo de regressão foi efetuada com base nos usuais critérios dos sinais esperados, significância dos estimadores, maximização do coeficiente de determinação e minimização da estatística de Schwarz.

(vi) Para evitar eventuais problemas de simultaneidade, foram tentadas especificações apenas com variáveis explicativas em períodos anteriores ao da variável explicada. Os hiatos, as expectativas e as dummies mencionadas foram incluídos alternativamente, enquanto o " $\mathrm{k}$ ” máximo para os hiatos e os choques cambiais foi igual a 12 .

Assim, o melhor modelo de regressão obtido para representar a curva de 
Phillips novo-keynesiana para o Brasil no período de abril de 2000 a outubro de 2008 foi o seguinte: ${ }^{45}$

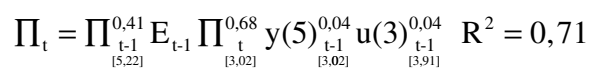

Com base na relação empírica (9), os seguintes resultados foram obtidos, para o período total da amostra utilizada:

Tabela 2

\begin{tabular}{|c|c|c|c|c|}
\hline \multicolumn{5}{|c|}{ DETERMINANTES DA INFLAÇÃO - RESULTADOS PARA O PERÍODO ABR/2000-OUT/2008 } \\
\hline & $\begin{array}{l}\text { Persistência } \\
\text { inflacionária }\end{array}$ & $\begin{array}{l}\text { Choques } \\
\text { cambiais }\end{array}$ & $\begin{array}{l}\text { Expectativas } \\
\text { de inflação }\end{array}$ & $\begin{array}{l}\text { Hiato de utilização } \\
\text { de capacidade }\end{array}$ \\
\hline Especificação & $\begin{array}{l}\text { Inflação do } \\
\text { mês anterior }\end{array}$ & $\begin{array}{l}\text { Desvalorização } \\
\text { média nos } \\
\text { três meses } \\
\text { anteriores }\end{array}$ & $\begin{array}{l}\text { Média das medianas } \\
\text { nos cinco primeiros } \\
\text { dias úteis do mês } \\
\text { anterior }\end{array}$ & $\begin{array}{l}\text { Média dos cinco } \\
\text { meses anteriores }\end{array}$ \\
\hline & 0,4137 & 0,0405 & 0,6828 & 0,0395 \\
\hline $\begin{array}{l}\text { Elastıcıdade da intlaçao } \\
\text { Coeficiente de variação }\end{array}$ & 0,0046 & 0,0274 & 0,0018 & 0,0100 \\
\hline Resposta da inflação & 0,0019 & 0,0011 & 0,0012 & 0,0004 \\
\hline Coef. Determinação Parcial & 0,5131 & 0,4521 & 0,3466 & 0,1171 \\
\hline
\end{tabular}

Assim, pode-se constatar que:

(i) A persistência inflacionária, embora não tendo a maior elasticidade nem a maior variabilidade, foi o principal determinante da inflação no período. Apresentou a maior resposta média a choques de um coeficiente de variação na própria inflação defasada e o maior coeficiente de determinação parcial, ${ }^{6}$ que indica que a persistência inflacionária explicou mais da metade das variações da inflação.

(ii) Os choques cambiais apresentaram a maior variabilidade e, apesar de sua reduzida elasticidade, foi o segundo principal determinante da inflação no período, explicando mais de $45 \%$ de suas variações, de acordo com o coeficiente de determinação parcial estimado.

(iii) As expectativas inflacionárias foram o determinante de maior elasticidade, o que garantiu uma influência relativamente importante sobre a inflação, apesar de ser o que apresentou a menor variabilidade.

\footnotetext{
${ }^{4}$ Os valores entre colchetes correspondem à estatística $t$.

${ }^{5}$ Os resíduos não possuem raízes unitárias, conforme testes apresentados na Tabela 1, indicando ausência de perturbações desta natureza na regressão estimada.

${ }^{6}$ Esses coeficientes estimam a parcela das variações na variável dependente explicada com a inclusão de determinada variável explicativa, mantendo-se as demais no modelo.
} 
(iv) hiato de capacidade foi o menos importante dos determinantes considerados, no período como um todo, apesar da segunda maior variabilidade, com menores elasticidade, resposta a seus choques e coeficiente de determinação.

Vale ressaltar que as estimativas geradas com a utilização de todo o período amostral de uma vez refletirão o comportamento médio em todo período, não captando suas alterações ao longo do tempo. Tais limitações foram superadas com a estimação de coeficientes de determinação parcial em períodos móveis dos últimos 36 meses ao longo de todo período amostral. Tais coeficientes estimam o poder explicativo de determinada variável, controlando-se os efeitos das demais. Os Gráficos 2 e 3 apresentam as trajetórias dos coeficientes de determinação parciais de cada determinante da inflação em períodos móveis dos últimos 36 meses, o que possibilita a verificação da importância relativa e absoluta de cada variável explicativa, em cada período e ao longo do tempo.

Avaliando-se os Gráficos 2 e 3, os resultados indicam que:

(i) Os choques cambiais estiveram entre os principais determinantes da inflação até o período móvel encerrado em maio de 2006, passando a ser pouco relevantes a partir de então, possivelmente em função de sua volatilidade relativamente moderada.

(ii) A persistência inflacionária foi mais importante que as expectativas durante quase todo o período considerado.

(iii) Embora o hiato de utilização de capacidade tenha apresentado influência moderada no comportamento da inflação ao longo de quase todo o período, nos últimos meses chegou a alcançar a maior importância relativa dentre os determinantes analisados.

Gráfico 2: Coeficiente de determinação parcial para a inflação

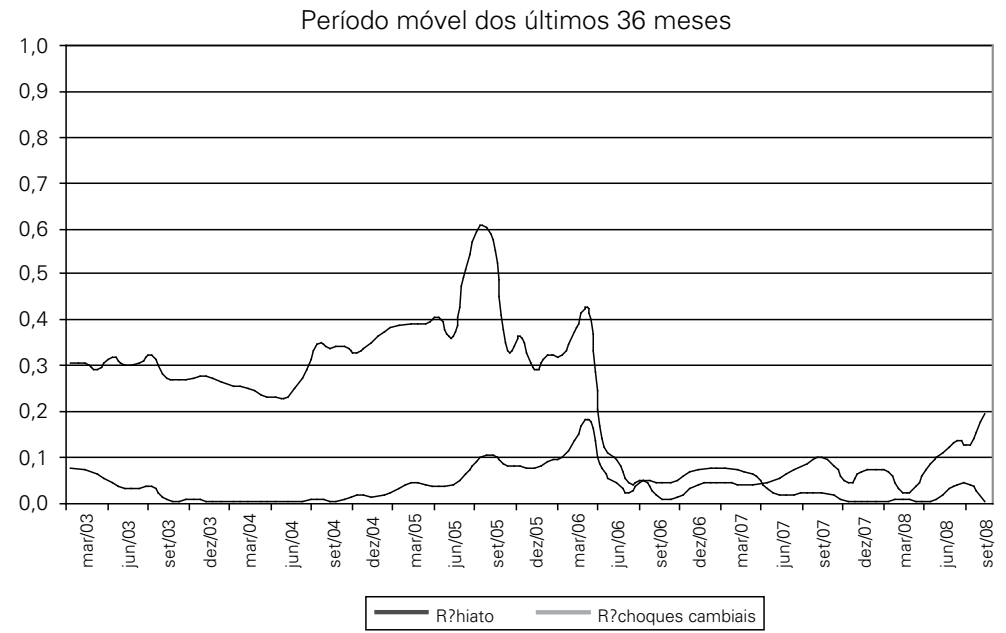


Gráfico 3: Coeficiente de determinação parcial para a inflação

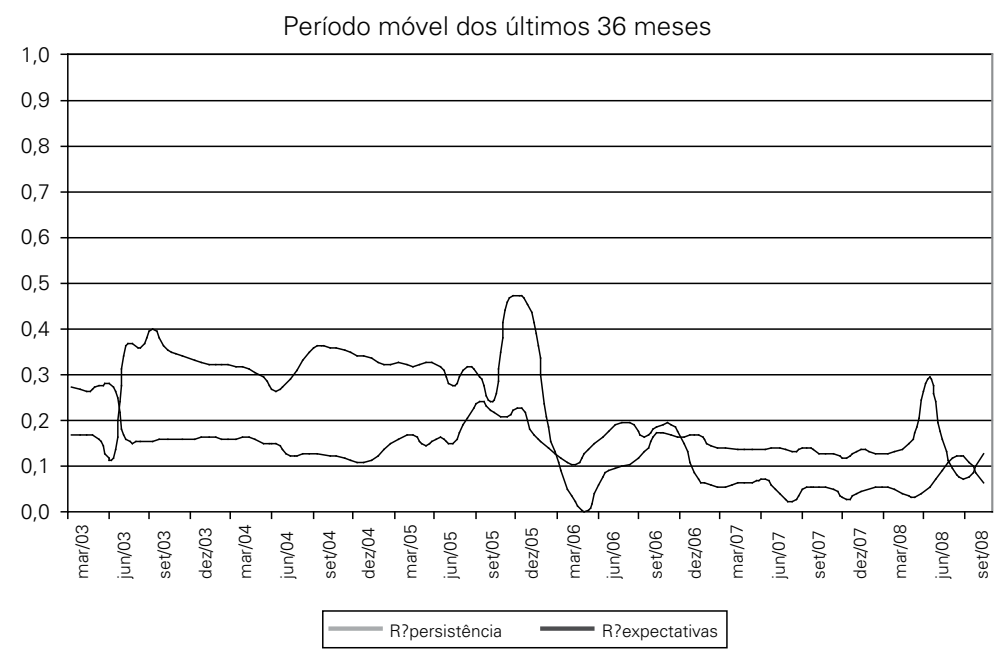

\section{DISCUSSÃO}

A literatura empírica sobre inércia inflacionária no Brasil é bastante extensa, mas não apresenta resultados consistentes entre os diversos trabalhos, o que reduz o interesse em um levantamento dos mesmos. Todavia, vale mencionar o trabalho de Maia e Cribari-Neto (2006), em que, embora os autores não se preocupem em diferenciar os conceitos de inércia e de persistência inflacionária, as técnicas aplicadas e o período considerado, após a implementação do Plano Real em 2004, estão mais relacionados ao que neste trabalho estamos denominando persistência.

Em termos de modelagem estatística, se uma série temporal possuir uma raiz unitária, então suas inovações apresentarão persistência ao longo do tempo. Entretanto, uma mesma série temporal pode se comportar como um processo de raiz unitária em certos períodos, nos quais apresentaria persistência, e como um processo estacionário em outros períodos. Em Maia e Cribari-Neto (2006), testou-se a presença de raiz unitária nas séries de variações mensais do IGP-DI e do IPCA, de agosto de 1994 a abril de 2004, tanto globalmente, com o teste de DickeyFuller aumentado (ADF), quanto para quantis selecionados, a partir de representações autorregressivas quantílicas (AQ).

Os resultados dos testes ADF indicam que inflação brasileira, de acordo com as séries e no período considerado, é globalmente estacionária, assim como nos resultados obtidos neste trabalho com os testes ADF e PP, aplicados ao IPCA do período de abril de 2000 a outubro de 2008, conforme Tabela 1. Contudo, os testes aplicados para diversos quantis revelam que o processo não alcança estacionaridade na cauda superior da distribuição condicional, indicando que os choques ocorridos em períodos de inflação mais elevada possuem tempo de dissipação su- 
perior, o que é compatível com a presença de persistência inflacionária, conforme modelado neste trabalho na forma apresentada em (9).

Dentre os trabalhos empíricos recentes sobre curva de Phillips para o Brasil, vale mencionar Lima (2003), Fasolo e Portugal (2004) e Schwartzman (2006). Com base em séries abrangendo o período de 1982 a 2001, Lima (2003) realiza estudo empírico sobre a NAIRU para a economia brasileira. Em função da instabilidade inflacionária no período, aquele autor utilizou duas técnicas para tratar possíveis quebras estruturais, introduzindo resíduos $\mathrm{ARCH}$ e adicionando cadeias de Markov para a variância. Como resultados, obteve evidências de que a NAIRU brasileira se alterou ao longo do tempo, apesar da estabilidade da inclinação da curva de Phillips, deixando de ser estatisticamente diferente da taxa de desemprego observada a partir do segundo semestre de 1995. Este último resultado, embora pouco valorizado pelo próprio autor, é consistente com os aqui obtidos com a curva de Phillips e coeficientes de determinação parcial estimados, que sugerem que o hiato do produto tem tido pouca importância relativa na determinação do comportamento da inflação brasileira, ao menos do início do atual regime de câmbio flutuante a meados de 2008.

Fasolo e Portugal (2004) baseiam suas análises na CPNK com rigidez de preços, tentando realizar um avanço no tratamento empírico dado nos últimos anos às expectativas inflacionárias no Brasil, em geral assumidas como racionais e representadas pelo valor futuro da própria série de inflação a ser modelada. Utilizando séries para o período de 1990 a 2002, aqueles autores se propõem a avaliar as relações entre inflação e suas expectativas usando um modelo de mudanças de regimes baseado em cadeias de Markov. Em seguida, CPNK é estimada usando filtro de Kalman, com as séries do exercício anterior mais a série de taxa de desemprego sazonalmente ajustada pelo método Census-X11 e de um indicador de assimetria de preços. Finalmente, estima-se um sistema de equações, por Full-Information Maximum Likelihood (FIML), com inflação, expectativas de inflação, variações da taxa Selic, M1 ajustado pelo Census-X11 e desvios da NAIRU como variáveis endógenas, e o indicador de assimetria de preços como variável exógena.

As expectativas de inflação são estimadas por um procedimento amplamente aceito, qual seja, extraí-la das taxas negociadas no mercado financeiro. Especificamente, os autores pretenderam utilizar as taxas dos CDB pré-fixados e pós-fixados para calcular as referidas expectativas. Contudo, as taxas pós-fixadas, disponíveis no local e para o período indicado pelos autores, não são para os CDB corrigidos por índices de preços, mas por TR e TBC. Lamentavelmente, este equívoco compromete os resultados empíricos obtidos, apesar das sofisticadas técnicas aplicadas.

Em Schwartzman (2006), curvas de Phillips são estimadas por mínimos quadrados de três estágios com preços desagregados em segmentos de bens comercializáveis, não comercializáveis e monitorados, com amostras de dados trimestrais iniciando-se em 1997, 1998 e 1999 e encerrando-se no terceiro trimestre de 2003. Também esse trabalho, mesmo com metodologia e amostras totalmente diferentes das aqui adotadas, reforça um importante resultado obtido. A importância relativamente bai- 
xa do hiato de capacidade, nas especificações obtidas em nossa pesquisa até meados de 2008, é consistente com a baixa significância da variável "utilização de capacidade" obtida por aquele autor. Apenas no segmento de bens não comercializáveis a utilização de capacidade foi significativa naquela pesquisa. A mais importante conclusão daquele trabalho, contudo, é baseada em testes de restrições aos parâmetros estimados das curvas de Phillips, que estabelecem a impossibilidade de rejeição da hipótese de verticalidade da curva de Phillips para o Brasil.

\section{CONCLUSÕES}

Este artigo apresenta uma fundamentação teórica para a persistência inflacionária com a autoridade monetária mantendo credibilidade e política adequadas e sem depender exclusivamente de indexação de preços. Tal fundamentação, em nível microeconômico, teria suporte na hipótese de racionalidade limitada de Simon. Nesse sentido, os modelos propostos em Mash (2006) e Sheedy (2007) obtêm formalmente tais resultados, que correspondem a relações macroeconômicas compatíveis com qualquer fundamentação microeconômica para a rigidez de preços que acomode a hipótese de probabilidade de ajustamento dos preços crescente com a idade de sua fixação. Uma hipótese complementar compatível seria que, na presença de mercados imperfeitos, agentes com suficiente poder de mercado tentariam elevar e manter suas margens de lucro recorrentemente, desequilibrando preços relativos fazendo com que todos os agentes tentem repassar os aumentos de custos provocados.

Especificamente em termos empíricos, as principais contribuições do trabalho seriam:

(1) A inclusão de expectativas inflacionárias efetivamente observadas, ao contrário de alguns trabalhos que assumem expectativas racionais e adotam valores futuros da variável dependente como indicador.

(ii) A identificação de uma série de hiato estatisticamente significante na especificação da curva de Phillips novo-keynesiana proposta, o que não foi alcançado em trabalhos mais recentes que abordaram as relações entre inflação e produto no Brasil, conforme discutido na quinta seção. ${ }^{7}$ Com a amostra aqui utilizada, que inclui observações mais recentes, a relação entre inflação e hiato de capacidade tornou-se suficientemente sistemática para ser especificada em um modelo clássico de regressão.

(iii) Identificação da relevância, absoluta e relativa, das variações defasadas sobre o comportamento de curtíssimo prazo da inflação corrente. Tais in-

\footnotetext{
${ }^{7}$ Tal dificuldade pode ter decorrido da maior importância relativa de outros determinantes nas amostras utilizadas, como os choques cambiais e a persistência inflacionária, como pode ser visualizado nos Gráficos 2 e 3.
} 
fluências revelariam a presença de persistência inflacionária, que pode vir sendo condicionada por indexação e outros mecanismos.

(iv) $\mathrm{O}$ uso de coeficientes de determinação parcial em períodos móveis dos últimos 36 meses ao longo de todo período amostral, que possibilita a verificação da importância relativa e absoluta de cada variável explicativa, em cada período e ao longo do tempo.

Com uma curva de Phillips novo-keynesiana, estimada para o Brasil no período 2000-2008, e os coeficientes de determinação parciais para subperíodos móveis de 36 meses se identifica a persistência inflacionária como o principal determinante da inflação. Os choques cambiais concorreram com a persistência até meados de 2006, possivelmente pela alta volatilidade ocorrida no período móvel considerado no cálculo dos coeficientes de determinação parciais. As expectativas mantiveram relevância no período, ainda que parte dela deva ser atribuída ao simples conhecimento do padrão sazonal da inflação. O hiato de capacidade só veio a apresentar maior importância para o comportamento da inflação ao final do período amostral, podendo sua reduzida influência anterior estar associada ao predomínio de outros determinantes.

Assim, pesquisas para identificar os determinantes da persistência, assim como sua importância no processo inflacionário, seriam de interesse para qualquer economia, mesmo as com baixo nível de indexação de preços. Em particular, nas economias cuja persistência inflacionária não pode ser classificada como residual, como seria o caso da brasileira, é especialmente relevante identificar os seus determinantes. Uma possível estratégia seria investigar os efeitos sobre os preços da indexação em setores de serviços públicos e alguns segmentos do mercado de trabalho, bem como de imperfeições nos mercados, sobretudo decorrentes de poder de mercado e falhas de regulação. ${ }^{8}$

Com relação à efetividade da política monetária, vale observar que a mesma não condiciona a persistência inflacionária, podendo apenas ter um efeito compensatório através dos demais determinantes. Nesse sentido, diante de uma insuficiente potência das expectativas na determinação da inflação, os processos de desinflação baseados apenas na política monetária parecem estar dependendo cada vez mais do canal de transmissão via taxa de câmbio e de hiatos de capacidade, o que dificulta o crescimento da economia

Portanto, a persistência inflacionária exige política monetária mais dura quando não se aceita acomodá-la nem se adotam medidas complementares para reduzila. Assim, assumindo-se a hipótese de a persistência de níveis inflacionários mais elevados resultar de componente inercial remanescente superior ao de economias desenvolvidas, a minimização dos mecanismos de indexação teriam efeitos favoráveis. Caso resultem de maiores imperfeições nos mercados, a recomendação direta

\footnotetext{
${ }^{8}$ Estudos empíricos sobre as influências dos níveis de mark-up sobre a inflação podem ser encontrados em Neiss (2001), Cavelaars (2003), Smets e Wouters (2003) e Przybyla e Roma (2005), que confirmam essa hipótese tanto para os países da OECD quanto para os da União Europeia.
} 
seriam políticas que visassem aumentar a competitividade e práticas mais efetivas e adequadas de regulação de setores pouco competitivos.

\section{REFERÊNCIAS BIBLIOGRÁFICAS}

AMATO, J. D.; LAUBACH, T. "Rule of thumb behavior and monetary policy". European Economic Review, 47:761-790, 2003.

BALL, L. Disinflation with imperfect credibility. "Journal of Monetary Economics" 35: 5-23, 1995. . Near Rationality and Inflation in Two Monetary Regimes. NBER Working Paper $n^{\circ} 7988$, 2000.

BALL, L.; MANKIW, N. G. "The NAIRU in theory and practice”. Journal of Economic Perspectives, 16: 115-136, 2002.

BARSKY, R. B. "The fisher effect and the forecastability and persistence of inflation". Journal of Monetary Economics, 19: 3-24, 1987.

BEGG, D. K. H.; IMPERATO I. The Rationality of Information Gathering: Monopoly. Manchester School, 69(3): 237-52, 2001.

BULLARD, J.; MITRA, K. “Learning about monetary policy rules”. Journal of Monetary Economics, 49(6): 1105-1129, 2002.

CAVELAARS, P. “Does competition enhancement have permanent inflation effects?” Kyklos, 56(1), 69-94, 2003.

CALVO, G. A. "Staggered prices in a utility maximizing framework”. Journal of Monetary Economics, 12: 383-398, 1983.

CARROLL, C. The Epidemiology of Macroeconomic Expectations. NBER Working Paper $n^{\circ} 8695$, 2001.

CHRISTIANO, L. J.; EICHENBAUM, M.; EVANS, C. "Nominal rigidities and the dynamic effects of a shock to monetary policy". Journal of Political Economy, 113: 1-45, 2005.

COGLEY, T.; SBORDONE, A. M. A Search for a Structural Phillips Curve. Federal Reserve Bank of New York staff report $n^{\circ} 203,2005$.

COLLARD, F.; DELLAS, H. The New Keynesian Model with Imperfect Information and Learning. IDEI Working Papers n ${ }^{\circ} 273$. University of Toulouse, 2004.

EVANS, G.; HONKAPOHJA, S. Learning and Expectations in Macroeconomics. Princeton: Princeton University Press, 2001.

FASOLO, A. M.; PORTUGAL M. S. "Imperfect rationality and inflationary inertia: a new estimation of the Phillips Curve for Brazil”. Estudos Econômicos, 34(4): 2004.

GORDON, R. J. The "time-varying Nairu and its implications for economic policy". Journal of Economic Perspectives, 11: 11-32, 1997.

HOLT, C. C.; MODIGLIANI, F.; MUTH J. F.; SIMON, H. A. Planning Production Inventories and Workforce Englewood Cliffs (N.J.): Prentice-Hall, 1960.

LIMA, E.C.R. The NAIRU, "Unemployment and the rate of inflation in Brazil". Revista Brasileira de Economia, 57(4): 899-930, 2003.

MAIA, A. L. S.; CRIBARI-NETO, F. "Dinâmica inflacionária brasileira: resultados de auto-regressão quantílica”. Revista Brasileira de Economia, 60(2): 153-165, 2006.

MANKIW, N. G. "Small menu costs and large business cycles: a macroeconomic model of monopoly". Quarterly Journal of Economics, 100: 529-537, 1985.

MANKIW, N. G.; REIS, R. "Sticky information versus sticky prices: A proposal to replace the new Keynesian Phillips Curve”. Quarterly Journal of Economics, 117(4): 1295-1328, 2002.

MASH, R. Optimising Microfoundations for Inflation Persistence. Computing in Economics and Finance 2006, $\mathrm{n}^{\circ}$ 457. Society for Computational Economics, 2006.

MILANI, F. Adaptive Learning and Inflation Persistence. University of California-Irvine, Department of Economics. Working Paper n ${ }^{\circ}$ 050607, 2005. 
NEISS, K. “The markup and inflation: evidence in OECD countries”. Canadian Journal of Economics, 34(2), 570-587, 2001.

NEWEY, W.; WEST K. "A simple positive semi-definite, heteroskedasticity and autocorrelation consistent covariance matrix”. Econometrica, 55, 703-708, 1987.

ORPHANIDES, A.; WILLIAMS J. C. Imperfect Knowledge, Inflation Expectations, and Monetary Policy. NBER Working Paper n 9884, 2003.

PALOVIITA, M. "Inflation dynamics in the Euro area and the role of expectations: Further results". Empirical Economics, 31(4), 847-860, 2006.

PRZYBYLA, M.; ROMA M. Does Product Market Competition Reduce Inflation? evidence from EU countries and sectors. ECB working paper series $n^{\circ} 453,2005$.

SARGENT, T. Bounded Rationality in Macroeconomics. New York: Oxford University Press, 1993.

SENT, E. M. Sargent versus Simon: bounded rationality unbound. Cambridge Journal of Economics, 21: 323-338, 1997.

SHEEDY, K. D. Intrinsic Inflation Persistence. CEP Discussion Paper ${ }^{\circ}$ 837. London School of Economics, 2007.

SIMON, H. A. The Sciences of the Artificial, $2^{\text {nd }}$ ed. Cambridge: MIT, 1981.

SIMONSEN, M. H. Price stabilization and income policies: Theory and the Brazilian case study. In: DORNBUSCH, R.; SIMONSEN, M. H., Eds. Inflation, Debt and Indexation. Cambridge: MIT Press, 1983.

SIMS, C. "Implications of rational inattention". Journal of Monetary Economics, 50(3): 497-720, 2003.

SCHWARTZMAN, F. F. Estimativa de Curva de Phillips para o Brasil com Preços Desagregados. Economia Aplicada, 10(1): 137-155, 2006.

SMETS, F.; WOUTERS, R. An estimated dynamic stochastic general equilibrium model of the Euro area. Journal of European Economic Association 1(5), 1123-1175: 2003.

WOODFORD, M. "Interpreting inflation persistence: comments on the conference on 'Quantitative evidence on price determination." Journal of Money, Credit and Banking, 39(1), 203-210, 2007.

ZBARACKI, M. J. et al. "The managerial and customer costs of price adjustment: Direct evidence from industrial markets”. The Review of Economics and Statistics, 86(2): 514-533, 2004. 